Präv Gesundheitsf 2021 · 16:296-302 https://doi.org/10.1007/s11553-020-00822-9

Eingegangen: 2. September 2020

Angenommen: 19. November 2020

Online publiziert: 16 . Dezember 2020

(c) Der/die Autor(en) 2020

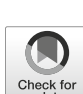

Torsten Michael Bollweg (D) - Orkan Okan (D) - Alexandra Frețian - Christina Janner • Tessa Schulenkorf · Sandra Kirchhoff · Paulo Pinheiro • Ullrich Bauer

Interdisziplinäres Zentrum für Gesundheitskompetenzforschung (IZGK), Fakultät für

Erziehungswissenschaft, Universität Bielefeld, Bielefeld, Deutschland

\title{
Dimensionen der Gesundheitskompetenz von Viertklässler*innen
}

\section{Ergebnisse einer explorativen Querschnittsstudie in Nordrhein-Westfalen}

\section{Zusatzmaterial online \\ Zusätzliche Informationen sind in der Online-Version dieses Artikels (https://doi. org/10.1007/s11553-020-00822-9) enthalten.}

Die Gesundheitskompetenz gerät als potenziell modifizierbare Determinante der Gesundheit zunehmend in den Fokus von Gesundheitsförderung und Prävention. Kinder und Jugendliche werden hierbei als zentrale Zielgruppe identifiziert, bislang ist jedoch nur wenig über die Gesundheitskompetenz junger Menschen bekannt. Dieser Beitrag stellt Daten zur Gesundheitskompetenz von Viertklässler*innen in Nordrhein-Westfalen vor und zeigt hierbei auf, wie schon im Alter von 9 bis 10 Jahren verschiedene Dimensionen der Gesundheitskompetenz entlang sozioökonomischer Merkmale ungleich verteilt sind.

\section{Hintergrund}

Gesundheitskompetenz (GK) kann als „das Wissen, die Motivation und die Fähigkeiten von Menschen [verstanden werden], relevante Gesundheitsinformationen [...] zu finden, zu verstehen, $\mathrm{zu}$ beurteilen und anzuwenden" [24]. Laut der Weltgesundheitsorganisation (WHO) ist GK eine „kritische Determinante der Gesundheit", die integraler Bestandteil der Fähigkeiten und Kom- petenzen sein muss, die insbesondere durch das schulische Curriculum vermittelt werden [28]. Damit wird die Förderung von GK klar in Kindheit und Jugend verortet. Bislang liegen jedoch kaum Daten vor, anhand derer die GK von Kindern und Jugendlichen beschrieben und Interventionsbedarfe identifiziert werden könnten. Dies gilt insbesondere dann, wenn nur Kinder in den Blick genommen werden (hier verstanden als Menschen im Alter von $<13$ Jahren), die für Ansätze der Primärprävention und Gesundheitsförderung eine besonders bedeutende Zielgruppe darstellen. Während Übersichtsarbeiten zwar immerhin 20 Studien identifizieren konnten, die verschiedene Aspekte der GK (u. a.) bei Kindern messen [10, 14, 15, 18] (für eine Übersicht s. [2]), sind es für Deutschland nur 3 Studien ${ }^{1}$ :

- Wallmann et al. [27] untersuchen gesundheitsbezogenes Wissen,

- Schmidt et al. [21] erheben Wissen, Kommunikation und Einstellungen und

- Bollweg et al. [3] erfassen selbstberichtete sowie funktionale GK.

Die von Bollweg et al. [3] gesammelten Daten wurden bislang nur hinsichtlich der Entwicklung eines Instruments zur

\footnotetext{
1 Noch nicht durch Literaturreviews erfasst ist zudem das von Teufl et al. [25] entwickelte Quiz zur Erhebung von GK bei Kindern (QUIGK-K), das ebenfalls die Wissensebene adressiert.
}

Erfassung der GK von Kindern ausgewertet [3, 4]. Im Rahmen dieses Beitrags erfolgt daher erstmalig eine inhaltliche Darstellung der gesammelten Daten im Bereich der selbstberichteten GK, des Gesundheitswissens, sowie gesundheitsbezogener schriftsprachlicher und numerischer Fähigkeiten von Viertklässler*innen, um potenzielle Forschungsund Interventionsbedarfe aufzuzeigen.

\section{Methode}

Es wurde eine einmalige, schriftliche Klassenraumbefragung an Schulen in Nordrhein-Westfalen (NRW) durchgeführt.

\section{Zielgruppe}

Zielgruppe waren Schüler*innen der vierten Grundschulklasse (Alter meist 9 bis 10 Jahre), da hier eine für eine standardisierte schriftliche Befragung ausreichende schriftsprachliche Kompetenz angenommen werden kann und diese junge Zielgruppe bisher kaum beforscht wurde.

\section{Rekrutierung}

Grundschulen im Umkreis von ca. $100 \mathrm{~km}$ um Duisburg, dem Sitz des durchführenden Umfragezentrums (Sozialwissenschaftliches Umfragezentrum [SUZ]), wurden zur Teilnahme eingeladen. Schulleiter*innen wurden pos- 
Präv Gesundheitsf 2021 · 16:296-302 https://doi.org/10.1007/s11553-020-00822-9

(c) Der/die Autor(en) 2020

\title{
T. M. Bollweg · O. Okan · A. Frețian · C. Janner · T. Schulenkorf · S. Kirchhoff · P. Pinheiro · U. Bauer \\ Dimensionen der Gesundheitskompetenz von Viertklässler*innen. Ergebnisse einer explorativen Querschnittsstudie in Nordrhein-Westfalen
}

\section{Zusammenfassung}

Hintergrund. Die Gesundheitskompetenz (GK) junger Menschen wird von Wissenschaft und Politik zunehmend als Ansatzpunkt für Gesundheitsförderung und Prävention anerkannt. Gerade die GK von Kindern wurde jedoch, insbesondere in Deutschland, bisher kaum erforscht. Diese Studie widmet sich dieser Forschungslücke, indem sie die GK von Viertklässler*innen in Nordrhein-Westfalen (NRW) untersucht.

Ziel der Arbeit (Fragestellung). Die Fragestellung ist die explorative Beschreibung verschiedener Dimensionen der GK von Kindern unter Berücksichtigung sozioökonomischer Merkmale.

Material und Methoden. Es wurde eine schriftliche Klassenraumbefragung durch- geführt. Der Fragebogen, der speziell für 9- bis 10-Jährige entwickelt wurde, umfasst drei Dimensionen der GK: selbstberichtete allgemeine GK, funktionale GK (schriftsprachliche und numerische Fähigkeiten) und das Gesundheitswissen.

Ergebnisse. Es wurden 899 Schüler*innen an 32 Schulen befragt. Hierbei wurde eine hohe selbstberichtete GK ermittelt: die Befragten empfinden den Umgang mit Gesundheitsinformationen als einfach. Kinder mit niedrigem Wohlstand schneiden in allen Dimensionen der GK signifikant schlechter ab. Dies gilt mit Ausnahme der selbstberichteten GK auch für Kinder, die zuhause nicht nur Deutsch sprechen.
Schlussfolgerung. Diese Studie untersucht erstmals die GK von Viertklässler*innen in NRW. Wenngleich die selbstberichtete GK hoch ist, zeigt sich schon bei dieser jungen Altersgruppe ein sozialer Gradient hinsichtlich Wohlstand und Sprache. Die Forschungslage ist für das Kindesalter zwar noch lückenhaft und es besteht weiterhin Forschungsbedarf, die Ergebnisse deuten aber auf einen frühen Interventionsbedarf hin, um allen Kindern unabhängig von ihrer Herkunft zu ermöglichen, GK in dem Maß zu entwickeln, wie es der eigenen Gesundheit zuträglich ist.

Schlüsselwörter

Gesundheitswissen $\cdot$ Kinder $\cdot$ Schule $\cdot$ Sozialer Gradient · Studie

\section{Fourth-graders' health literacy. Results from an explorative cross-sectional study in North Rhine-Westphalia, Germany}

\begin{abstract}
Background. The health literacy $(\mathrm{HL})$ of young people is increasingly being acknowledged by research and policy as an entry point for health promotion and prevention. However, there is little research on children's health literacy, particularly in Germany. This study addresses this research gap by investigating fourth-graders' HL in the German federal state of North Rhine-Westphalia (NRW).

Objectives. To explore the extent of children's $\mathrm{HL}$ taking into consideration socioeconomic characteristics.

Materials and methods. A classroom-based pen-and-paper survey was conducted.

The questionnaire, which was developed specifically for 9-to 10-year-olds, addresses

three dimensions of HL: self-reported general $\mathrm{HL}$, functional $\mathrm{HL}$ (health-related literacy and numeracy), and health knowledge.

Results. Data were collected from 899 students visiting 32 schools. A high level of self-reported HL was observed: participants reported that it was easy for them to deal with health information. Children that had the lowest family affluence scored significantly lower in all dimensions of $\mathrm{HL}$. With the exception of self-reported $\mathrm{HL}$, this also applies for children that report a home language other than German.

Conclusions. This is the first study to investigate fourth-graders' HL in NRW, Germany. Although self-reported HL is high,
\end{abstract}

a social gradient related to family affluence and home language can already be observed in this young age group. While the available evidence is inconclusive and more research is needed, our results suggest that there is a need for early prevention to ensure that all children, irrespective of their background, can develop a level of $\mathrm{HL}$ that helps promote their health.

\section{Keywords}

Health knowledge $\cdot$ Child · Schools · Social gradient - Surveys and questionnaires talisch über die Erhebung informiert, woraufhin Erziehungsberechtigte um die informierte Einwilligung zur Teilnahme ihres Kindes gebeten wurden.

\section{Datenerhebung}

Die schriftliche Klassenraumerhebung wurde zwischen November 2016 und Mai 2017 von geschulten Interviewer ${ }^{\star}$ innen des SUZ durchgeführt.

\section{Erhebungsinstrumente}

Die GK wurde multidimensional erhoben, d. h. mit Hinblick auf selbstberichtete (allgemeine) GK, funktionale GK sowie gesundheitsbezogenes Wissen. Ferner wurden soziodemografische Merkmale erfragt. Die genutzten Items finden sich in Anhang 1 (Zusatzmaterial online), weiterführende Angaben zur Auswertung in Anhang 2 (Zusatzmaterial online).

\section{Selbstberichtete (allgemeine) Gesundheitskompetenz}

Der HLS-Child-Q15, eine altersadaptierte Version des HLS-EU-Q [11], wurde eingesetzt, um die empfundene Schwierigkeit des Umgangs mit gesundheitsbezogenen Informationen („selbstberichtete allgemeine $\mathrm{GK}^{“}$ ) $\mathrm{zu}$ erfassen. Die Fragen beginnen mit „Wie einfach oder schwierig ist es für dich, ... ?" und werden auf einer 4-stufigen Ratingskala von 1 - „sehr schwierig“ bis 4 - „sehr einfach“ 


\begin{tabular}{|c|c|c|c|}
\hline Geschlecht & $n$ & \multicolumn{2}{|l|}{$\%$} \\
\hline Mädchen & 480 & \multicolumn{2}{|l|}{53,4} \\
\hline Jungen & 404 & \multicolumn{2}{|l|}{44,9} \\
\hline Fehlend & 15 & \multicolumn{2}{|l|}{1,7} \\
\hline Alter & MW & \multicolumn{2}{|l|}{ SD } \\
\hline Gesamt & 9,56 & \multicolumn{2}{|l|}{0,62} \\
\hline- & $n$ & \multicolumn{2}{|l|}{$\%$} \\
\hline 8 & 6 & \multicolumn{2}{|l|}{0,7} \\
\hline 9 & 427 & \multicolumn{2}{|l|}{47,5} \\
\hline 10 & 412 & \multicolumn{2}{|l|}{45,8} \\
\hline 11 & 41 & \multicolumn{2}{|l|}{4,6} \\
\hline 12 & 4 & \multicolumn{2}{|l|}{0,4} \\
\hline Fehlend & 9 & \multicolumn{2}{|l|}{1,0} \\
\hline Mit Eltern gesprochene Sprache & $n$ & \multicolumn{2}{|l|}{$\%$} \\
\hline Nur Deutsch & 578 & \multicolumn{2}{|l|}{64,3} \\
\hline Mindestens eine andere Sprache & 302 & \multicolumn{2}{|l|}{33,6} \\
\hline Fehlend & 19 & \multicolumn{2}{|l|}{2,1} \\
\hline Materieller Wohlstand & MW & \multicolumn{2}{|l|}{ SD } \\
\hline Gesamt & 8,65 & \multicolumn{2}{|l|}{2,33} \\
\hline- & $n$ & \multicolumn{2}{|l|}{$\%$} \\
\hline $0-5$ & 83 & 9,4 & \multirow[t]{2}{*}{ Niedrigster Wohlstanc } \\
\hline 6 & 81 & 9,0 & \\
\hline 7 & 92 & 10,2 & \multirow[t]{4}{*}{ Mittlerer Wohlstand } \\
\hline 8 & 139 & 15,5 & \\
\hline 9 & 144 & 16,0 & \\
\hline 10 & 140 & 15,6 & \\
\hline 11 & 103 & 11,5 & \multirow[t]{3}{*}{ Höchster Wohlstand } \\
\hline 12 & 70 & 7,8 & \\
\hline 13 & 27 & 3,0 & \\
\hline Fehlend & 20 & \multicolumn{2}{|l|}{2,2} \\
\hline
\end{tabular}

beantwortet. Zudem wird die Kategorie „weiß nicht“ angeboten. Die Entwicklung und statistische Überprüfung des HLSChild-Q15, die ebenfalls mit dem hier beschriebenen Datensatz vollzogen wurde, wurde anderswo beschrieben [3, 4, 13]. Die Skala weist eine gute interne Konsistenz auf (Cronbachs $\alpha=0,791$; [3]).

\section{Funktionale Gesundheitskompetenz}

Zur Erfassung schriftsprachlicher Fähigkeiten („literacy“) im Kontext Gesundheit („funktionale GK“) wurde ein in Anlehnung an den „test of functional health literacy in adults" (TOFHLA; [17]) entwickelter Lückentext eingesetzt. Hierbei wurden in fünf Sätzen zum Thema Impfung 12 Wörter entfernt, für die aus je

\section{Soziodemografische Daten}

(FAS III [26]) eingesetzt, welche mit sechs Items den materiellen familiären Wohlstand erfasst und seit 2013/14 als Standardinstrument der „Health Behaviour in School-aged Children"(HBSC)Studie eingesetzt wird. Folgenden Gruppen wurden gebildet: „niedrigster Wohlstand“ (1. Quintil), „mittlerer Wohlstand“ (2. bis 4. Quintil) und „höchster Wohlstand“ (5. Quintil).

Als Proxy für einen Migrationshintergrund wurde die mit den Eltern gesprochene Sprache erfasst. Die Antworten wurden zusammengefasst als „nur Deutsch“ und „mindestens eine andere Sprache“.

\section{Statistische Analyse}

Die Beschreibung der Daten erfolgt deskriptivstatistisch anhand absoluter und relativer Häufigkeiten, Mittelwerten und Standardabweichungen. Unterschiede hinsichtlich Alter, Geschlecht, Wohlstand und der zuhause gesprochenen Sprache wurden mittels Mann-Whitney- $U$-Test auf statistische Signifikanz geprüft, wobei ein $p$-Wert von $<5 \%$ angelegt wurde.

\section{Ergebnisse}

\section{Stichprobe}

An 37 von 200 eingeladenen Schulen $(18,5 \%)$ konnten $n=1037$ Schüler ${ }^{*}$ innen befragt werden. Hiervon entfallen jedoch fünf Schulen und $n=130$ Schüler ${ }^{*}$ innen auf ein Pretest-Sample zur Erprobung von Skalen und Antwortformaten. Ferner wurden $n=8$ Schüler ${ }^{\star}$ innen von der Analyse ausgeschlossen, da z. B. die Teilnahme abgebrochen wurde. Insgesamt konnten die Daten von 899 Schüler*innen herangezogen werden, verteilt auf 32 Schulen und 65 Klassen. 53,4\% sind weiblich und das Durchschnittsalter beträgt 9,56 Jahre $(S D=0,62)$. Fast zwei Drittel spricht zuhause nur Deutsch. Die Stichprobenmerkmale sind in • Tab. 1 dargestellt.

\section{Selbstberichtete allgemeine Gesundheitskompetenz}

Als Proxy für den sozioökonomischen Status wurde die Family Affluence Scale
Der Mittelwert für die Stichprobe liegt bei 3,34 ( $\mathrm{SD}=0,37$; s. - Tab. 2), der 


\begin{tabular}{|c|c|c|c|c|c|}
\hline Selbst. Gesundheitskompetenz & $n$ & $\%$ & MW & SD & Min-Max \\
\hline Gesamt & 844 & 93,6 & 3,34 & 0,37 & $1,86-4,00$ \\
\hline \multicolumn{6}{|l|}{ Nach Quintilen } \\
\hline 1. - niedrigste GK in Stichprobe & 185 & 21,9 & 2,81 & 0,24 & $1,86-3,07$ \\
\hline 2. bis 4. - mittlere GK & 480 & 56,9 & 3,38 & 0,16 & $3,07-3,64$ \\
\hline 5. - höchste GK in Stichprobe & 179 & 21,2 & 3,80 & 0,10 & $3,67-4,00$ \\
\hline \multicolumn{6}{|l|}{ GK-Level gemäß HLS-EU-Q } \\
\hline „Inadäquate GK“ (0-50 \% max. Score) & 21 & 2,5 & 2,30 & 0,18 & $1,86-2,50$ \\
\hline „Problematische GK" (50-66\%) & 106 & 12,6 & 2,79 & 0,12 & $2,53-2,93$ \\
\hline „Ausreichende GK“ (66-84\%) & 390 & 46,2 & 3,26 & 0,15 & $3,00-3,47$ \\
\hline „Exzellente GK“ ( $\geq 84 \%)$ & 327 & 38,7 & 3,70 & 0,14 & $3,50-4,00$ \\
\hline \multicolumn{6}{|c|}{ "Literacy“ (schriftsprachliche Fähigkeiten; Lückentext) } \\
\hline Gesamt & 874 & 97,2 & 9,73 & 1,98 & $0-12$ \\
\hline 0-5 richtig ergänzte Begriffe & 31 & 3,5 & - & - & - \\
\hline 6 & 31 & 3,5 & - & - & - \\
\hline 7 & 43 & 4,9 & - & - & - \\
\hline 8 & 84 & 9,6 & - & - & - \\
\hline 9 & 126 & 14,4 & - & - & - \\
\hline 10 & 189 & 21,6 & - & - & - \\
\hline 11 & 229 & 26,2 & - & - & - \\
\hline 12 richtig ergänzte Begriffe & 141 & 16,1 & - & - & - \\
\hline \multicolumn{6}{|l|}{ "Numeracy“ (Rechenaufgaben) } \\
\hline Gesamt & 836 & 91,0 & 1,98 & 0,87 & $0-3$ \\
\hline 0 Aufgaben richtig gelöst & 42 & 5,0 & - & - & - \\
\hline 1 & 201 & 24,0 & - & - & - \\
\hline 2 & 324 & 38,8 & - & - & - \\
\hline 3 Aufgaben richtig gelöst & 269 & 32,2 & - & - & - \\
\hline \multicolumn{6}{|l|}{ Wissen } \\
\hline Gesamt & 896 & 99,7 & 2,60 & 1,12 & $0-5$ \\
\hline 0 Fragen richtig beantwortet & 13 & 1,5 & - & - & - \\
\hline 1 & 143 & 16,0 & - & - & - \\
\hline 2 & 273 & 30,5 & - & - & - \\
\hline 3 & 275 & 30,7 & - & - & - \\
\hline 4 & 150 & 16,7 & - & - & - \\
\hline 5 Fragen richtig beantwortet & 42 & 4,7 & - & - & - \\
\hline MW Mittelwert, SD Standardabweichung & & & & & \\
\hline
\end{tabular}

Umgang mit Gesundheitsinformationen wird als „eher einfach“ bis „sehr einfach“ empfunden. Es lassen sich keine Unterschiede nach Geschlecht, Alter oder der mit den Eltern gesprochenen Sprache nachweisen. Jedoch erzielen Kinder mit dem niedrigsten materiellen Wohlstand ( $\mathrm{MW}=3,24 ; \mathrm{SD}=0,43)$ schlechtere Werte als Kinder mit mittlerem $(\mathrm{MW}=3,36$; $\mathrm{SD}=0,36 ; p<0,05)$ und hohem Wohlstand $(\mathrm{MW}=3,39 ; \mathrm{SD}=0,32 ; p<0,01)$. Kinder mit mittlerem und hohem Wohlstand unterscheiden sich nicht signifikant.

\section{Funktionale \\ Gesundheitskompetenz}

Der Durchschnittswert im Bereich „literacy“ beträgt 9,73 $(\mathrm{SD}=1,98)$ von maximal 12 richtigen Antworten. Es lassen sich keine signifikanten Unterschiede nach Alter und Geschlecht beobachten. Kinder mit dem niedrigsten Wohlstand haben jedoch schlechtere Werte als Kinder mit mittlerem (9,27 vs. 9,76; $p<0,01)$ oder dem höchsten Wohlstand (9,27 vs. $10,08 ; p<0,001)$. Auch Kinder mit mittlerem und hohem Wohlstand unterschei- lassen sich Unterschiede in Abhängigkeit von der mit den Eltern gesprochenen Sprache beobachten („nur Deutsch“ vs. „mindestens eine andere Sprache“: 10,07 vs. 9,13; $p<0,001)$.

Im Bereich „numeracy“ erzielten $5,0 \%$ der Schüler ${ }^{*}$ innen keine, $24,0 \%$ eine, $38,8 \%$ zwei und $32,2 \%$ drei richtige Antworten. Im Durchschnitt wurden zwei Aufgaben richtig beantwortet $(\mathrm{MW}=1,98 ; \mathrm{SD}=0,87)$. Es lassen sich keine Unterschiede nach Alter und Geschlecht beobachten. Schüler*innen mit dem niedrigsten Wohlstand erzielen schlechtere Werte als solche mit mittlerem $(1,71$ vs. 2,$01 ; p<0,01)$ oder dem höchsten Wohlstand (1,71 vs. 2,12 ; $p<0,001)$. Schüler*innen mit mittlerem und dem höchsten Wohlstand unterscheiden sich nicht signifikant. Zuletzt erzielen auch Schüler*innen, die zuhause nur Deutsch sprechen, bessere Werte $(2,1$ vs. 1,$77 ; p<0,001)$.

\section{Gesundheitsbezogenes Wissen}

Im Durchschnitt wurden 2,59 $(\mathrm{SD}=1,12)$ von fünf Wissensfragen richtig beantwortet. Es wurden keine Unterschiede nach Alter und Geschlecht festgestellt. Schüler ${ }^{\star}$ innen mit dem niedrigsten Wohlstand weisen niedrigere Werte auf als solche mit mittlerem (2,29 vs. 2,66 ; $p<0,001)$ oder dem höchsten Wohlstand (2,29 vs. 2,$74 ; p<0,001)$. Schüler ${ }^{*}$ innen mit mittlerem und hohem Wohlstand unterscheiden sich nicht signifikant. Schüler*innen, die nur Deutsch mit ihren Eltern sprechen, erreichen höhere Werte als solche, die auch eine andere Sprache sprechen $(2,7$ vs. 2,$41 ; p<0,01)$. den sich (9,76 vs. 10,$08 ; p<0,05)$. Zudem

\section{Dimensionen der Gesundheits- kompetenz - Zusammenhänge}

Selbstberichtete GK korreliert mit keiner der anderen Dimensionen der GK in statistisch signifikanter Hinsicht („literacy“: $p=0,065$; „numeracy“: $p=0,144$; Wissen: $p=0,340)$. „Literacy“ korreliert dahingegen mit "numeracy“ $(\mathrm{r}=0,298 ; p<0,01)$ und Gesundheitswis$\operatorname{sen}(\mathrm{r}=0,184 ; p<0,01)$ und auch „numeracy“ und Gesundheitswissen korrelieren signifikant $(r=0,146 ; p<0,01)$. 


\section{Diskussion}

\section{Stichprobe}

Erwartungsgemäß sind die meisten Teilnehmer*innen 9 oder 10 Jahre alt ( $n=839 ; 93,3 \%$ der Stichprobe), es haben jedoch auch $8-(n=6 ; 0,7 \%), 11$ $(n=41 ; 4,6 \%)$, und 12-Jährige $(n=4$; $0,4 \%)$ teilgenommen. Dies wirkt zunächst überraschend, ist jedoch mit der gängigen Schulpraxis zu erklären, die die Einschulung mit 5 Jahren, das Überspringen einer Schulklasse, die Rückstellung von der Einschulung sowie die Wiederholung eines Schuljahres ermöglicht. Eine separate Betrachtung der 8- und 12-Jährigen scheint zwar ratsam, ist aufgrund der geringen Fallzahl (insgesamt $n=10$ ) hier jedoch fraglich. Bei der Betrachtung der 11-Jährigen, die mit $n=41$ stärker vertreten sind, zeigt sich, dass diese gegenüber den 9- und 10Jährigen schlechter abschneiden. Diese Unterschiede in selbstberichteter GK, Gesundheitswissen und schriftsprachlicher Fähigkeiten sind gegenüber den 9- und 10-Jährigen signifikant, bei den Rechenfertigkeiten nur gegenüber den 10-Jährigen. Dieser Befund ist kontraintuitiv, da bei älteren Schüler ${ }^{*}$ innen ein besseres Kompetenzniveau erwartet werden kann. Hier kommt jedoch vermutlich der Umstand zum Tragen, dass nicht 11-Jährige in ihrer „regulären“ Klassenstufe (5. oder 6. Klasse) mit 9- und 10-Jährigen in der 4. Klasse verglichen werden, sondern eben diejenigen 11-Jährigen, die aus verschiedenen Gründen „noch“ die 4. Klasse besuchen. Daher wurden alle Altersgruppen in die Analysen einbezogen, um keine idealtypische Klasse abzubilden, sondern die vorliegende Stichprobe in ihrer Gesamtheit zu beschreiben.

\section{Selbstberichtete allgemeine Gesundheitskompetenz}

Die Mehrheit der befragten Kinder empfindet kaum Schwierigkeiten im Umgang mit Gesundheitsinformationen. Selbst das Quintil mit der geringsten GK erreicht mit 2,81 noch einen Mittelwert im leicht positiven Bereich (vgl. - Tab. 2). Werden die Schwellenwerte der HLS-
EU-Q-Studie [11] angelegt, haben 84,9\% eine „ausreichende“ (46,2\%) oder ,,exzellente“ GK (38,7\%), während für 15,1\% eine „problematische“ (12,6\%) oder „inadäquate“ GK $(2,5)$ festgestellt wird (s. - Tab. 2). Da der HLS-EU-Q zuvor nicht für das Kindesalter adaptiert wurde, liegen keine Referenzwerte vor. Für Erwachsene in NRW sowie Jugendliche in Österreich wurde allerdings ein höherer Anteil mit "problematischer“ oder „inadäquater“ GK ermittelt (NRW: 46,3\% [23]; AT: 58,0\% [19]). Der direkte Vergleich ist aber nur eingeschränkt möglich, da sich HLS-Child-Q15 und HLS-EU-Q in Inhalt und Struktur unterscheiden.

Der Großteil der im Kindesalter durchgeführten Studien kann im Bereich der selbstberichteten, allgemeinen $G K$ nicht zum Vergleich herangezogen werden, da z. B. funktionale GK, Wissen, Einstellungen oder spezifische Inhalte wie die psychische Gesundheit und Asthma erfasst werden (vgl. [2]). Brown et al. [5] berichten jedoch, dass $74,3 \%$ der 9-jährigen und 85,6\% der 10-jährigen Befragten das meiste, was sie über Gesundheit hören, laut Selbstauskunft (sehr) einfach verstehen können. Dies lässt sich als hohe selbstberichtete GK interpretieren, die auch hier beobachtet wurde.

Kinder, die einen niedrigen Wohlstand aufweisen, empfinden den Umgang mit Gesundheitsinformationen als schwieriger. Dieser soziale Gradient konnte bereits im Erwachsenenalter beobachtet werden [23] und auch im Jugendalter lässt sich ein Gradient mit Hinblick auf den Wohlstand - nicht jedoch die Sprache - beobachten [19]. Auch Manganello et al. [12] beobachten Unterschiede hinsichtlich der Ethnizität und des Wohlstands, nicht aber hinsichtlich der gesprochenen Sprache. Diese Befunde decken sich mit unseren Daten. Für einen bzgl. der Altersgruppe direkten Vergleich hinsichtlich des Gradienten selbstberichteter, allgemeiner GK liegen bislang jedoch keine Daten vor.

\section{Zur Interpretation selbstberichte- ter Gesundheitskompetenz}

Bei der Interpretation der Befunde ist die Relationalität des Konstrukts GK zu berücksichtigen [16]: Während „Gesundheitskompetenz" begrifflich auf ein individuelles Attribut verweist, entfaltet sich selbstberichtete $G K$ als Abwägen zwischen wahrgenommenen Bewältigungsressourcen und Anforderungen. Die empfundene Einfachheit dabei, „herauszufinden, welches Essen für dich gesund ist" (HLS-Child-Q15 Item 4) spricht z.B. zunächst nur dafür, dass Kinder den Eindruck haben, die Herausforderungen bewältigen zu können, denen sie bei der Wahl von Lebensmitteln begegnen. $\mathrm{Ob}$ dieses Urteil durch ein starkes Selbstvertrauen, gutes Wissen über Nahrungsmittel, die Unterstützung durch Bezugspersonen oder durch Selbstüberschätzung zustande kommt, ergibt sich nur mittelbar. Vor diesem Hintergrund muss auch diskutiert werden, warum z. B. jüngere Menschen vermeintlich eine bessere Gesundheitskompetenz aufweisen [20], wenn aufgrund ihrer geringeren Erfahrung das Gegenteil zu erwarten wäre.

Selbstberichtete GK ist folglich ein subjektives und relationales $\mathrm{Maß}$, das nicht als individuelle Kompetenz interpretiert werden sollte. Die GK-Level der HLS-EU-Q-Studie („problematische GK“ usw.) sind daher kritisch zu betrachten - und werden hier deshalb berichtet - da sie potenziell fehlende Bewältigungs- und Unterstützungsressourcen begrifflich als Kompetenzdefizite auslegen. Gerade im Kindesalter darf die Bedeutung des sozialen Netzwerks bei der Bewältigung gesundheitsbezogener Anforderungen nicht unterschätzt werden, wobei im Diskurs die Einbettung der GK in soziale Gefüge bislang noch nicht ausreichend berücksichtigt wird [1].

\section{Funktionale \\ Gesundheitskompetenz}

In dieser Studie ließ sich ein deutlicher sozialer Gradient der „literacy“ und „numeracy“ belegen. Dieser Gradient konnte auch in früheren Studien beobachtet werden, z. B. als Zusammenhang zwi- 
schen der Ethnizität der Teilnehmenden, ihrer „literacy“ $[6,12]$ und „numeracy“ [12]. Auch Unterschiede hinsichtlich des Wohlstands und der zuhause gesprochenen Sprache wurden beschrieben [12]. Dem stehen jedoch Studien gegenüber, die keine solche Unterschiede berichten $[8,22]$. Die Studienlage ist somit wenig eindeutig. Dennoch weisen unsere Daten darauf hin, dass schriftsprachliche und numerische gesundheitsbezogene Fähigkeiten schon im Grundschulalter ungleich verteilt sind.

\section{Gesundheitsbezogenes Wissen}

Auch im Bereich Gesundheitswissen wurde ein sozialer Gradient beobachtet. Ähnliche Ergebnisse liefern Studien, die ein besseres Gesundheitswissen unter Kindern von Eltern mit höherem Bildungsgrad [21] sowie unter Gymnasiast*innen nachweisen [27]. Darüber hinaus mangelt es jedoch auch in diesem Bereich an Evidenz.

\section{Dimensionen der Gesundheits- kompetenz}

Zwar hängt selbstberichtete (allgemeine) GK theoretisch mit grundlegenden Formen der GK zusammen, dies wurde hier aber nicht bestätigt: In unserer Stichprobe ist das Urteil darüber, ob der Umgang mit gesundheitsbezogenen Informationen als schwierig oder einfach empfunden wird, nicht von funktionalen Fähigkeiten und Wissen abhängig. Eine mögliche Erklärung ist, dass die gesundheitsbezogenen Bereiche, die im HLS-Child-Q15 erfragt werden, mithilfe sozialer Unterstützungsinstanzen und Informationsangebote bewältigt werden können, ohne dass auf funktionale GK und Wissen zurückgegriffen werden muss. Möglich ist jedoch auch, dass die Bereiche, in denen Kinder ihre eigenen Kompetenzen anwenden, um mit Gesundheitsinformationen umzugehen, nicht durch den Fragebogen erfasst wurden.

Auch zu beachten ist, dass nur bivariate Zusammenhänge dargestellt wurden, die das komplexe Zusammenspiel zwischen den Variablen kaum abbilden. In multivariaten Analysen, die Frețian et al.
[9] mit den hier beschriebenen Daten vollzogen haben, zeigte sich unter Kontrolle weiterer Variablen z.B. eine signifikante Beziehung zwischen Literacy und selbstberichteter GK. Zur Beziehung funktionaler und selbstberichteter GK im Kindesalter ist daher weitere Forschung nötig.

In anderen Studien, die mittels HLSEU-Q selbstberichtete GK erfassen, zeigt sich, dass bei 15- [19] sowie 14- bis 17Jährigen [7] selbstberichtete und funktionale GK korrelieren, was auch bei Erwachsenen beobachtet wurde [11].

\section{Materieller Wohlstand}

In dieser Studie kam die FAS III zur näherungsweisen Erfassung des materiellen Wohlstands zum Einsatz. Die FAS ist insbesondere dann hilfreich, wenn Standardindikatoren wie das Familieneinkommen sowie der Beruf und Bildungsabschluss der Eltern nicht verlässlich erfasst werden können - was auch hier der Fall war, da nur Kinder selbst befragt wurden. Bei der Interpretation der mittels FAS gesammelten Befunde ist jedoch zu beachten, dass die Skala die ökonomische Lebenssituation der Befragten nur mittelbar widerspiegelt. Primär werden materielle Güter im Haushalt adressiert, wobei z. B. eine geringe Anzahl von Autos oder Computern im Familienbesitz als Indikator für einen geringen Wohlstand interpretiert wird. Ein ausgeprägtes Nachhaltigkeitsdenken oder der gezielte Verzicht - beides keineswegs notwendigerweise mit Armut verbunden - werden so möglicherweise als geringer Wohlstand fehlinterpretiert. Dementsprechend ist es ratsam, dass der hier beschriebene Gradient der GK in zukünftigen Studien auch mit alternativen Indikatoren ergründet wird, die den sozioökonomischen Status detaillierter erfassen und z.B. auch das subjektive Armutsempfinden adressieren.

\section{Limitationen}

Den Analysen liegt keine repräsentative Stichprobe zugrunde, weshalb die Generalisierbarkeit eingeschränkt ist. Zudem wurde die Befragung im Klassenraum durchgeführt, was sich ungünstig auf die Tendenz zum sozial erwünschten Antwortverhalten ausgewirkt haben kann. Eine Selbstüberschätzung im Bereich der selbstberichteten GK ist daher möglich. Ferner kamen in dieser Studie neu entwickelte Instrumente zum Einsatz, deren psychometrische Güte nicht durchgängig festgestellt wurde. Dem wäre der ausschließliche Einsatz validierter Instrumente vorzuziehen.

\section{Schlussfolgerung}

Dies ist die erste Studie, die verschiedene Dimensionen der GK bei Kindern in NRW erfasst. Die selbstberichtete GK der befragten Viertklässler*innen ist hoch: Sie empfinden den Umgang mit Gesundheitsinformationen als einfach. Dennoch lässt sich schon in dieser jungen Altersgruppe ein sozialer Gradient in allen Dimensionen der GK nachzeichnen: Materiell schlechter gestellte Kinder empfinden den Umgang mit Gesundheitsinformationen als schwieriger. Zudem schneiden sie schlechter in den Bereichen „literacy“, „numeracy“ und Gesundheitswissen $\mathrm{ab}$ - was auch für Kinder gilt, die zuhause (auch) eine andere Sprache als Deutsch sprechen. Diese Ergebnisse legen nahe, dass schon im Grundschulalter wesentliche Voraussetzungen für den kompetenten Umgang mit Gesundheitsinformationen nicht allen Kindern zur Verfügung stehen. Hier besteht folglich Forschungsbedarf bzgl. der Entstehungsbedingungen und Wechselwirkungen der verschiedenen Dimensionen der GK, da die GK von Kindern nach wie vor lückenhaft erforscht ist und auch international kaum Vergleichswerte vorliegen. Perspektivisch zeichnet sich jedoch auch deutlicher Interventionsbedarf $a b$, wenn von der Wichtigkeit der Gesundheitskompetenz für die Ermöglichung gesundheitlicher Partizipation und größtmöglicher Gesundheitschancen ausgegangen wird. Primäres Ziel sollte hierbei sein, dass alle Kinder unabhängig von ihrer sozialen und ökonomischen Herkunft ein optimales Maß an GK entwickeln können. 


\section{Fazit für die Praxis}

\section{- Schon im Grundschulalter ist die Gesundheitskompetenz von Kin- dern entlang sozioökonomischer Merkmale ungleich verteilt. \\ - Interventionen müssen daher früh ansetzen, um die Vergrößerung von Disparitäten der Gesundheitskompe- tenz im weiteren Lebensverlauf zu vermeiden. \\ - Mehr Forschung ist notwendig, um die Mechanismen der Entwicklung von Gesundheitskompetenz besser verstehen und Interventionsmöglich- keiten identifizieren zu können.}

\section{Korrespondenzadresse}

\section{Torsten Michael Bollweg}

Interdisziplinäres Zentrum für Gesundheitskompetenzforschung (IZGK), Fakultät für Erziehungswissenschaft, Universität Bielefeld Universitätsstraße 25, 33615 Bielefeld, Deutschland torsten.bollweg@uni-bielefeld.de

Förderung. Diese Arbeit wurde im Rahmen des vom Bundesministerium für Bildung und Forschung (BMBF; Förderkennzeichen: 01EL1424A 01EL1824A) geförderten Forschungsverbunds Health Literacy in Childhood and Adolescence (HLCA) durchgeführt (www.hlca-consortium.de).

Funding. Open Access funding enabled and organized by Projekt DEAL.

\section{Einhaltung ethischer Richtlinien}

Interessenkonflikt. T.M. Bollweg, O. Okan, A. Frețian, C. Janner, T. Schulenkorf, S. Kirchhoff, P. Pinheiro und U. Bauer geben an, dass kein Interessenkonflikt besteht.

Für diesen Beitrag wurden von den Autor*innen keine Studien an Menschen oder Tieren durchgeführt. Für die aufgeführten Studien gelten die jeweils dort angegebenen ethischen Richtlinien. Diese Studie wurde von der Ethikkommission der Universität Bielefeld (Referenznummer 2016-141-R) genehmigt. Die Teilnahme war freiwillig und nur nach informierter Einwilligung einer erziehungsberechtigten Person möglich.

Open Access. Dieser Artikel wird unter der Creative Commons Namensnennung 4.0 International Lizenz veröffentlicht, welche die Nutzung, Vervielfältigung Bearbeitung, Verbreitung und Wiedergabe in jeglichem Medium und Format erlaubt, sofern Sie den/die ursprünglichen Autor(en) und die Quelle ordnungsgemäß nennen, einen Link zur Creative Commons Lizenz beifügen und angeben, ob Änderungen vorgenommen wurden.
Die in diesem Artikel enthaltenen Bilder und sonstiges Drittmaterial unterliegen ebenfalls der genannten Creative Commons Lizenz, sofern sich aus der Abbildungslegende nichts anderes ergibt. Sofern das betreffende Material nicht unter der genannten Creative Commons Lizenz steht und die betreffende Handlung nicht nach gesetzlichen Vorschriften erlaubt ist, ist für die oben aufgeführten Weiterverwendungen des Materials die Einwilligung des jeweiligen Rechteinhabers einzuholen.

Weitere Details zur Lizenz entnehmen Sie bitte der Lizenzinformation auf http://creativecommons.org/ licenses/by/4.0/deed.de.

\section{Literatur}

1. Bauer U (2019) The social embeddedness of health literacy. In: Okan O, Bauer U, Levin-Zamir D et al (Hrsg) International handbook of health literacy. Research, practice and policy across the lifespan. Policy Press, Bristol, UK, S573-586

2. Bollweg TM, Okan O (2020) Gesundheitskompetenz messen bei Kindern: aktuelle Ansätze und Herausforderungen. In Bollweg TM, Bröder J, Pinheiro P (Hrsg) Health Literacy im Kindes- und Jugendalter. Springer Fachmedien Wiesbaden, Wiesbaden, $573-98$

3. Bollweg TM, Okan O, Fretian AM et al (2020) Adapting the European Health Literacy Survey Questionnaire for Fourth-Grade Students in Germany: Validation and psychometric analysis. Health Lit Res Pract 4(3):e144-e159

4. Bollweg TM, Okan O, Pinheiro P et al (2020) Adapting the European Health Literacy Survey for Fourth-Grade Students in Germany: Questionnaire development and qualitative pretest. Health Lit Res Pract 4(2):e119-e128

5. Brown SL, Teufel JA, Birch DA (2007) Early adolescents perceptions of health and health literacy. JSch Health 77(1):7-15

6. Davis TC, Wolf MS, Arnold CL et al (2006) Development and validation of the Rapid Estimate of Adolescent Literacy in Medicine (REALMTeen): a tool to screen adolescents for belowgrade reading in health care settings. Pediatrics 118(6):e1707-e1714

7. Domanska OM, Bollweg TM, Loer A-K et al (2020) Development and psychometric properties of a questionnaire assessing self-reported generic health literacy in adolescence. IJERPH 17(8):2860

8. Driessnack M, Chung S, Perkhounkova E et al (2014) Using the "newest vital sign" to assess health literacy in children. J Pediatr Health Care 28(2):165-171

9. Frețian A, Bollweg TM, Okan Oetal (2020) Exploring Associated Factors of Subjective Health Literacy in School-Aged Children. IJERPH 17(5):1720

10. Guo S, Armstrong R, Waters E et al (2018) Quality of health literacy instruments used in children and adolescents: A systematic review. BMJ Open 8(6):e20080

11. HLS-EU Consortium (2014) Comparative report on health literacy in eight EU member states. The European health literacy survey HLS-EU (Second extended and revised version, date July 22th, 2014)

12. Manganello JA, DeVellis RF, Davis TC et al (2015) Development of the Health Literacy Assessment Scale for Adolescents (HAS-A). J Commun Healthc 8(3):172-184

13. Okan O, Bollweg TM (2018) Development process of a health literacy measurement instrument for fourth grade schoolchildren: a mixed-method study. In Mayer A-K (Hrsg) Health Literacy across the Life Span. Pabst Science Publishers, Lengerich, S87-110

14. Okan O, Lopes E, Bollweg TM et al (2018) Generic health literacy measurement instruments for children and adolescents: a systematic review of the literature. BMCPublic Health 18(1):166

15. Ormshaw MJ, Paakkari L, Kannas L (2013) Measuring child and adolescent health literacy. A systematic review of literature. Health Educ 113(5):433-455

16. Parker R, Ratzan SC (2010) Health literacy: a second decade of distinction for Americans. J Health Commun 15(Suppl 2):20-33

17. Parker RM, Baker DW, Williams MV et al (1995) The test of functional health literacy in adults. A new instrument for measuring patients' literacy skills. JGen Intern Med 10(10):537-541

18. Perry EL (2014) Health literacy in adolescents: an integrative review. J Spec Pediatr Nurs 19(3):210-218

19. Röthlin F, Pelikan J, Ganahl K (2013) Die Gesundheitskompetenz von 15-jährigen Jugendlichen in Österreich. Abschlussbericht der österreichischen Gesundheitskompetenz Jugendstudie im Auftrag desHauptverbands derösterreichischen Sozialversicherungsträger (HVSV). https://lbihpr.lbg.ac.at/ en/oesterreichischen-gesundheitskompetenzjugendstudie.Zugegriffen:04.Dez. 2020

20. SchaefferD,VogtD, BerensE-Metal (2016) Gesundheitskompetenz der Bevölkerung in Deutschland. Ergebnisbericht. Universität Bielefeld, Fakultät für Gesundheitswissenschaften, Bielefeld

21. Schmidt CO, Fahland RA, Franze M et al (2010) Health-related behaviour, knowledge, attitudes, communication and social status in school children in Eastern Germany. Health Educ Res 25(4):542-551

22. Sharifl, BlankAE(2010) Relationship between child health literacy and body mass index in overweight children. Patient Educ Couns 79(1):43-48

23. Sørensen K, Pelikan JM, Röthlin F et al (2015) Health literacy in Europe. Comparative results of the European Health Literacy Survey (HLS-EU). Eur JPublic Health 25(6):1053-1058

24. Sørensen K, van den Broucke S, Fullam Jet al (2012) Health literacy and public health: a systematic review and integration of definitions and models. BMC Public Health 12:80

25. Teufl L, Vrtis D, Felder-Puig R (2019) QUIGK-K: Quiz zur Erhebung von Gesundheitskompetenz bei Kindern. Praev Gesundheitsf 3:282

26. Torsheim T, Cavallo F, Levin KA et al (2016) Psychometric validation of the Revised Family Affluence Scale: a latent variable approach. Child Indic Res 9:771-784

27. Wallmann B, Gierschner S, Froböse I (2012) Gesundheitskompetenz. Was wissen unsere Schüler über Gesundheit? (Health literacy. What do our students know about health?). Praev Gesundheitsf 7(1):5-10

28. WHO (2017) Shanghai declaration on promoting health in the 2030 Agenda for Sustainable Development. Health Promot Int 32(1):7-8

29. Zentrum für Kinder- und Jugendmedizin Heidelberg Medizin-fuer-kids.de - Was ist eine Impfung? http://www.medizin-fuer-kids.de/ kinderarztpraxis/kinderkrankheiten/impfung. htm. Zugegriffen: 17. Aug. 2020 